\title{
Evaluation of the scattered radiation components produced in a gamma camera using Monte Carlo method
}

\author{
Ivón Oramas Polo*
}

\begin{abstract}
Introduction: This paper presents a simulation for evaluation of the scattered radiation components produced in a gamma camera PARK using Monte Carlo code SIMIND. It simulates a whole body study with MDP (Methylene Diphosphonate) radiopharmaceutical based on Zubal anthropomorphic phantom, with some spinal lesions. Methods: The simulation was done by comparing 3 configurations for the detected photons. The corresponding energy spectra were obtained using Low Energy High Resolution collimator. The parameters related with the interactions and the fraction of events in the energy window, the simulated events of the spectrum and scatter events were calculated. Results: The simulation confirmed that the images without influence of scattering events have a higher number of valid recorded events and it improved the statistical quality of them. A comparison among different collimators was made. The parameters and detector energy spectrum were calculated for each simulation configuration with these collimators using ${ }^{99 \mathrm{~m}} \mathrm{Tc}$. Conclusion: The simulation corroborated that LEHS collimator has higher sensitivity and HEHR collimator has lower sensitivity when they are used with low energy photons.
\end{abstract}

Keywords Collimators, Monte Carlo, Gamma camera, SIMIND, Zubal phantom.

\section{Introduction}

In a gamma camera an event is considered valid when gamma ray is emitted perpendicular to the collimator holes, passes through a hole and interacts photo electrically in the Sodium Iodide activated with Thallium ( $\mathrm{NaI}(\mathrm{Tl})$ ) crystal, depositing all of its energy at a single location (Sorenson et al., 2002).

It is considered detector scatter event when gamma ray is emitted perpendicular to the collimator holes, passes through a hole and interacts by Compton scattering in the $\mathrm{NaI}(\mathrm{T} 1)$ crystal.

The scattered gamma ray can either interact a second time in the detector, in which case the full energy of gamma ray is deposited, or, it might escape the detector, in which case only part of the gamma ray energy is deposited. In the former case, energy discrimination cannot be used to reject the event, and the event will be mispositioned between the two interaction locations. In the latter case, it is likely that the event will be rejected because it does not satisfy the event energy criteria established by the upper and lower level discriminators (Sorenson et al., 2002).

The object scatter event is when the gamma ray is not emitted toward the collimator holes but is scattered by the body and passes through a collimator hole and subsequently is detected. The gamma ray loses energy during scattering and will therefore produce a smaller signal in the detector. Some of these events will be rejected by energy discrimination, but if the angle of scatter is small, the energy loss is small and the event may be accepted. In this case the event is mispositioned, often many centimeters from the original site of emission (Sorenson et al., 2002).

The event known as septal penetration is when a gamma ray is emitted toward the collimator, but not perpendicular to it. Because of incomplete attenuation by the thin collimator walls (septal penetration), there is a finite chance that the gamma ray will reach the $\mathrm{NaI}(\mathrm{T} 1)$ crystal and interact with it. This again leads to blurring of the image, because all events are considered to have come from a direction perpendicular to the collimator face. This effect becomes increasingly important when using high energy gamma emitters and/or high-resolution collimators with thin septa (Sorenson et al., 2002).

Only a first event provides correct positional information. Considerable effort is expended in the design of gamma cameras to reduce or eliminate the detection of the others events. The last three events are a cause of blurring and/or a loss of contrast in the image. Collimators also are carefully designed for specific energies to minimize septal penetration while maximizing sensitivity for a given gamma ray energy (Sorenson et al., 2002). 
Typically, a gamma camera is equipped with several interchangeable collimators. The differences among the collimators are the thickness, number and size of the holes and the way they are arranged or oriented. This, in turn, has an effect on the camera sensitivity, image magnification and image blur. The user must be aware of these differences in order to select the best collimator for a given examination (Sprawls, 2000).

When selecting a collimator, it is necessary to consider the energy of the gamma photons. The ability of a photon to penetrate a given material generally increases with photons energy. In other words, it takes a thicker piece of material to absorb high energy photons than it does to absorb low energy photons. The purpose of collimator septa is to prevent photons from penetrating from one hole to another. This depends on the relationship of the photon energy to the thickness of the metal septa separating the holes. With low energy photons, relatively thin septa are adequate. The advantage of thin septa is that holes can be located in a given area and this results in a higher sensitivity (Sprawls, 2000).

All the elements discussed above have a significant influence on the formation of the image and as a consequence in the clinical practice of nuclear medicine; so it is necessary to know how they affect the operation of the gamma camera. Choosing the correct collimator for desired study requires an understanding of the parameters that affect its performance.

The Monte Carlo (MC) method has been particularly useful in the development of scatter and attenuation correction methods, mainly due to its ability to differentiate between scattered and primary events in the image. Comparing simulated projection data without scattering and attenuation effects with scatter and attenuation corrected data can give the accuracy of the activity recovery. Investigations of both the shape of the scatter response functions and the fraction of scatter in the images can be made using MC calculations, providing valuable information for the development of scatter correction methods (Wernick and Aarsvold, 2004). Studies have been carried out for ${ }^{201} \mathrm{Tl}$ (Frey and Tsui, 1994) and (Kadrmas et al., 1998, 1999). Recently, studies have also been reported on ${ }^{131} \mathrm{I}$ (Dewaraja et al., 2000) and ${ }^{67} \mathrm{Ga}$ (De Vries et al., 1998). Comparative studies have been performed by several investigators (Buvat et al., 1995) and (Ljungberg et al., 1994).

De Vries et al. (1998) have studied the effects of collimator penetration, scatter, and $\mathrm{Pb} \mathrm{X}$-rays, which degrade the contrast, image resolution, and quantification of the images.

Monte Carlo techniques were extensively used to analyze the performance of new collimators design for planar scintillation cameras, SPECT and PET imaging. Selective comparisons among different collimators could also be presented for illustrative and teaching purposes (Zaidi, 2006).

The objective of this work is the evaluation of the scattered radiation components produced in a gamma camera PARK using Monte Carlo code SIMIND (Simulating Nuclear Medical Imaging Detectors) (SIMIND). In addition, a comparison among several collimators is also described.

\section{Methods}

The simulation of patient-like activity and tissue density distributions using analytical functions may be difficult, because it is not easy to model accurately the shapes of organs and structures in the body based on simple geometries. An alternative approach is, thus, to use digital images of a phantom. The relative location of each pixel determines its position in the $\mathrm{x}, \mathrm{y}$ and $\mathrm{z}$ directions, and the pixel value determines the activity, that is, the number of particles emitted from that location. Directly measured data from, for example, computed tomography $(\mathrm{CT})$ scanners can be used to allow the simulation of patients (Wernick and Aarsvold, 2004). An example is the Zubal phantom (Zubal et al., 1994). To construct the phantom, important organs and structures in the images were segmented and each of these regions is assigned a number so that each voxel can be identified as being part of a specific organ. This byte-coded phantom can be used to define tissue density and activity distributions in three dimensions. Computer phantom is flexible while providing accurate modeling of patient (Wernick and Aarsvold, 2004). The purpose of the phantom is to represent the organ or tissue of interest, to allow the radionuclide of interest to be contained in a specific volume and to provide a medium that absorbs and scatters the radiation emitted in a manner similar to tissue (Zaidi and Sgouros, 2002). Anthropomorphic phantoms with internally segmented structures make clinically realistic MC simulations possible.

In this paper it was simulated a whole body study with the radiopharmaceutical MDP based on the extended Zubal phantom with multiple spine lesions by SIMIND code. The simulation was done by comparing 3 configurations for the detected photons: The study without interactions with the phantom, the study with interactions with the phantom and the study with interactions with the phantom and backscattering material. For the evaluation of gamma camera collimators sensitivity the following were simulated: Low Energy High Resolution - LEHR, 
Low Energy Ultra High Resolution - LEUR, General Purpose Low Energy - LEGP), Low Energy High Sensitivity - LEHS), General Purpose Medium Energy - MEGP) and High Energy High Resolution - HEHR). The studies were simulated with the energy of $140 \mathrm{KeV}$, corresponding to Tc $99 \mathrm{~m}$, with $20 \%$ of energy windows for each collimators. The source used was the Zubal whole-body voxel man source distribution that is user- written source routine. This source routine provided the coordinates of a sampled decay position through the parameter list.

The MC simulation code SIMIND, developed by Professor Michael Ljungberg, describes a standard clinical SPECT camera and can easily be modified for almost any type of calculation or measurement encountered in SPECT imaging (SIMIND).

The SIMIND system has two main programs, named CHANGE and SIMIND. The CHANGE program provides a way of defining the system parameters to be simulated and writing data to external data files. The actual MC simulation is made by the program SIMIND that reads input files created by CHANGE and outputs results to screen or to different data files.
The CHANGE program enables the user to easily define the desired imaging system. CHANGE contains a series of menus that prompt the user to input parameters specific to the description of the system. These parameters are then written to a data file used in SIMIND. Table 1 shows some of the parameters used for the simulation.

The SIMIND was configured to simulate dual-head gamma camera PARK. The backscattering material is Lucite and the cover material is Aluminum. A large data base is included in CHANGE and it covers most of the commercial collimators available for both SPECT and planar imaging. However, the PARK collimators weren't included. The author included the PARK collimators into data base. It takes into account the shape and length of the holes, the septal distance (distance between two holes) and the physical width of collimators. Figure 1 shows a section of the database which includes the corresponding gamma camera PARK collimators on SIMIND. For the simulation of each collimator a code name must be given at the prompt of CHANGE to include a specific collimator. At this point, the new collimator parameters are calculated.

Table 1. Parameters for gamma camera simulation.

\begin{tabular}{|c|c|c|}
\hline \multicolumn{3}{|c|}{ General parameters for simulation } \\
\hline Parameter number & Parameter name & Parameter definition \\
\hline 9 & File phantom soft tissue & $\mathrm{h} 2 \mathrm{o}$ \\
\hline 10 & File phantom bone tissue & bone \\
\hline 11 & File cover material & al \\
\hline 12 & File crystal material & nai \\
\hline 13 & File densitymap & vox_man \\
\hline 14 & File sourcemap & vox_man \\
\hline 15 & File backscatter material & lucite \\
\hline \multicolumn{3}{|c|}{ Gamma camera parameters } \\
\hline 1 & Photon energy $(\mathrm{KeV})$ & 140 \\
\hline 8 & Crystal: Half /Length radius $(\mathrm{cm})$ & 29 \\
\hline 9 & Crystal: Thickness (cm) & 0.950 \\
\hline 10 & Crystal: Half width $(\mathrm{cm})$ & 21.500 \\
\hline 11 & Backscattering material: Thickness $(\mathrm{cm})$ & 3 \\
\hline 13 & Thickness of cover: $(\mathrm{cm})$ & 0.200 \\
\hline 20 & $\begin{array}{l}\text { Upper windows threshold }(\mathrm{KeV}) \text {. Instead of using the absolute values of } \\
\text { the upper threshold, a relative window definition can be given by using } \\
\text { a negative sign }\end{array}$ & -20 \\
\hline 21 & $\begin{array}{l}\text { Lower windows threshold }(\mathrm{KeV}) \text {. Instead of using the absolute values of } \\
\text { the lower threshold, a relative window definition can be given by using } \\
\text { a negative sign. }\end{array}$ & -20 \\
\hline 22 & Energy resolution $[140 \mathrm{KeV}](\%)$ & 13 \\
\hline 23 & Intrinsic resolution $[140 \mathrm{KeV}](\mathrm{cm})$ & 0.456 \\
\hline 26 & Number of photon histories * 1E6 & 10000 \\
\hline 27 & $\mathrm{KeV} /$ Channels & 1 \\
\hline 80 & Energy spectra channels & 512 \\
\hline
\end{tabular}




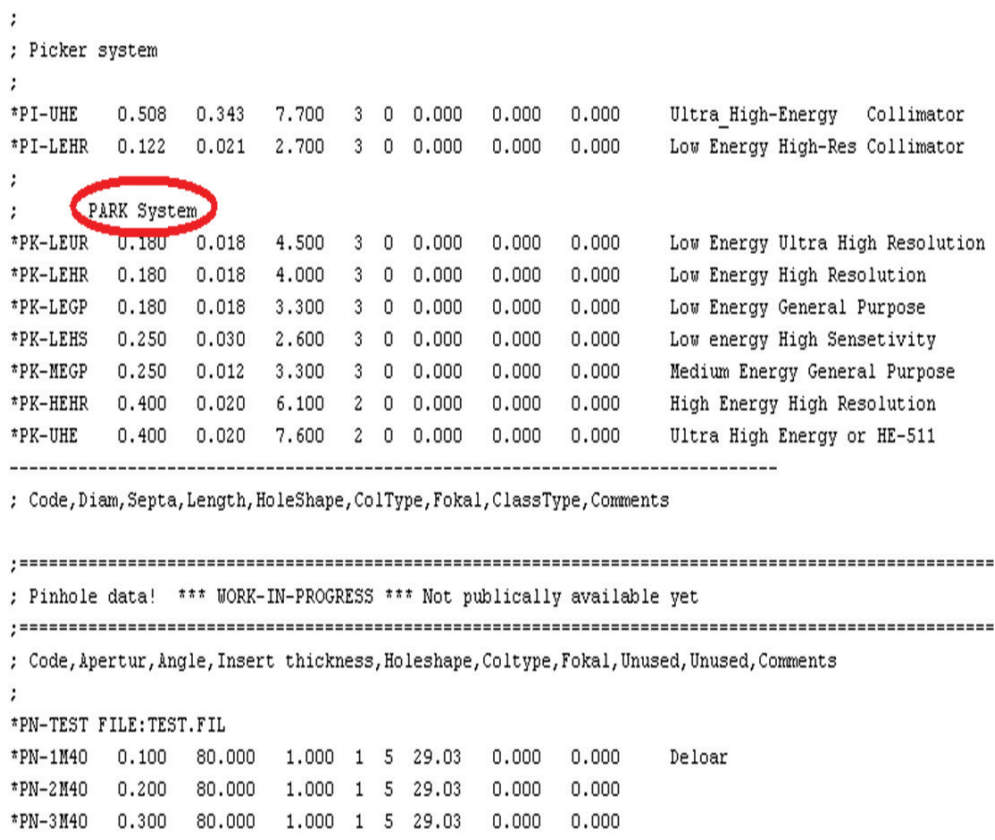

Figure 1. Section of the database which includes the corresponding collimators of gamma camera PARK in SIMIND.

The scintillation process was simulated for generation of photons of $140 \mathrm{keV}$ within the $\mathrm{NaI}$ (Tl) crystal. A source emitting photons with this energy was assigned to Tc $99 \mathrm{~m}$. An energy window was centered on the Tc $99 \mathrm{~m}$ photopeak (126-154 keV). The energy spectra created by SIMIND contains a spectrum of 512 channels. The first channel is the $\mathrm{keV} /$ channel while the others represent the actual energy distribution. The source was simulated for $37 \mathrm{MBq}$ of activity and at $25 \mathrm{~cm}$ from the detector surface. The energy spectra were displayed using Origin 6 program. The images were acquired with matrices of $192 \times 512$ pixels and a pixel size of $0.5 \mathrm{~mm}$.

SIMIND calculates some basic detector parameters by defaults. These appear on the screen and in the result file together with the input parameters, defined in CHANGE. Also the scatter fraction and percentage scatter are calculated. The calculated detector parameters are (SIMIND):

- Compton Area (Spectrum): The integral of simulated event from the first channel to the lower energy window channel $(126 \mathrm{keV})$. For energy $140 \mathrm{keV}$, the energy of the scattered photon depends on the angle of scatter. Photons that scatter in the forward direction loose very little energy in the scattering interaction and have energies very close to $140 \mathrm{keV}$. The statistical fluctuation within the detector makes some of them appear to have energies greater than $140 \mathrm{keV}$. The fluctuations within the detector cause the overlap between the scatter component and the photopeak of the spectrum. Photons that scatter in the backward direction $\left(180^{\circ}\right)$ have the lowest energy. For $140 \mathrm{keV}$ primary photons, complete backscatter produces $90 \mathrm{keV}$ photons. This means that scattered radiation produced by a $140 \mathrm{keV}$ primary source has photons energies ranging from $90 \mathrm{keV}$ to $140 \mathrm{keV}$. However, some photons may undergo two or more Compton interactions before leaving the body and this creates some photons with energies well below $90 \mathrm{keV}$. If a $140 \mathrm{keV}$ photon undergoes a single Compton interaction in the crystal, the maximum energy it can deposit is $50 \mathrm{keV}$. This occurs when the photon is scattered back out of the crystal $\left(180^{\circ}\right)$ and carries energy of $90 \mathrm{keV}$. Photons that scatter in a more forward direction have higher energies and therefore deposit less energy in the crystal. If characteristic X ray photon is created within the crystal, a different type of spectrum component is created. The energy deposited in the crystal is the difference between the energy of the primary photon and the escaping characteristic $\mathrm{X}$ ray photon. In $\mathrm{NaI}$ (Tl) crystal, the predominant characteristic $\mathrm{X}$ ray is the $28 \mathrm{keV}$ iodine $\mathrm{X}$ ray (Sprawls, 2000).

- Photo Area (Spectrum): The integral of simulated events between the lower energy 
window channel and the upper energy window channel $(154 \mathrm{keV})$. The photopeak of the spectrum results from the complete absorption of the gamma photons in the crystal by the photoelectric process. However, some of the photons that have been scattered in the patient with a small deflection angle (small loss of energy) will therefore have a possibility to be detected within the energy window and thus contribute to the image formation, but these photons carry wrong spatial information about the decay location in the object (Khalil, 2011).

- Pileup Area (Spectrum): The integral of simulated events from the upper energy window channel to the highest channel in the energy spectra.

- Fraction Photo (Window): This is the fraction of events in the energy window that originates from photons that have been completely absorbed in the crystal.

- Fraction Compton (Window): This is the fraction of events in the energy window that originates from Compton scattered photons.

- Scatter/Primary Ratio: This is the ratio between the events from photons scattered in the phantom to events from photons that have penetrated the phantom without interaction.

- Scatter/Total Ratio: This is the ratio between the events from photons scattered in the phantom to all events from photons.

- Percentage 1st order: This is the percentage of the scatter events originated from photons that have been scattered once in the phantom.

- Peak/Compton (Peak): The peak to Compton ratio may sometimes be defined as the maximum in the photo peak divided by the content in a typical channel below the Compton edge.

- Peak/Compton (Area): The integral counts in the energy window divided by the integral of counts below the lower energy threshold (or the Compton area).

- Peak/Total: The integral counts in the energy window divided by the integral of counts in the whole energy pulse- height distribution.

SIMIND calculates the energy spectra using program BIS. The main purpose of BIS the generation of two column ASCII string of the spectra to be used for plotting or printing.

For visualization and processing of the images obtained by the simulation, it was used the ImageJ program (version 1.45), developed by Wayne Rasband at the National Institute of Mental Health (NIMH)
Bethesda, Maryland, USA (Rasband and Ferreira, 2010). It is designed with an open architecture through "plugins" of Java. The acquisition, analysis and processing plugins can be developed using ImageJ's editor and Java compiler. The user-written plugins make it possible to resolve any type of processing and image analysis. The source code of the program is free. For visualization of the simulation resulting images nuclear medicine plugins were used. The obtained images are gray scale images. The gray scale images were changed by 16 color images for a better visualization of all structures of the images and details of the lesions.

\section{Results}

The scattered radiation components produced in a gamma camera PARK was evaluated. For this, three different SIMIND program configurations using LEHR collimator were simulated. Figure 2 shows the simulated images of study without interactions with the phantom and their corresponding energy spectrum. The main gamma ray interactions with the detector were by photoelectric absorption and Compton scattering. Most of the photoelectric interactions resulted in a full deposition of the energy in the detector. In the energy spectrum it is observed a photopeak at location corresponding to the energy $140 \mathrm{KeV}$ of ${ }^{99 \mathrm{~m}} \mathrm{Tc}$. In the case of Compton scattering, only a part of gamma ray energy was transferred to the detector through the Compton recoil electron. The scattered escaping gamma rays deposited their energy into the detector and this was about $50 \mathrm{KeV}$. The peaks have been broader due to the poor energy resolution of the camera.

Because of the contribution of scattered photons is small, there is an image with good contrast and resolution. All lesions were observed on the phantom.

In Figure 3, are shown the images with phantom interactions as well as the spectrum.

In Figure 4, are shown the images with interactions with the phantom and backscattering material behind crystal. Also, its spectrum is shown.

The images of Figures 3 and 4 show the degradation due to the influence of scattering in the phantom that simulates the patient, scattering from the photomultiplier tubes (PMT). The general effect of scattering is the addition of events in the lower energy region of spectrum. The spectra for these simulations show an enlargement in comparison to the spectrum shown in Figure 2. This is a result of the registration of scatter events. These events lead to a loss of contrast and resolution in the images. When comparing Figures 3 and 4 with Figure 2, it 

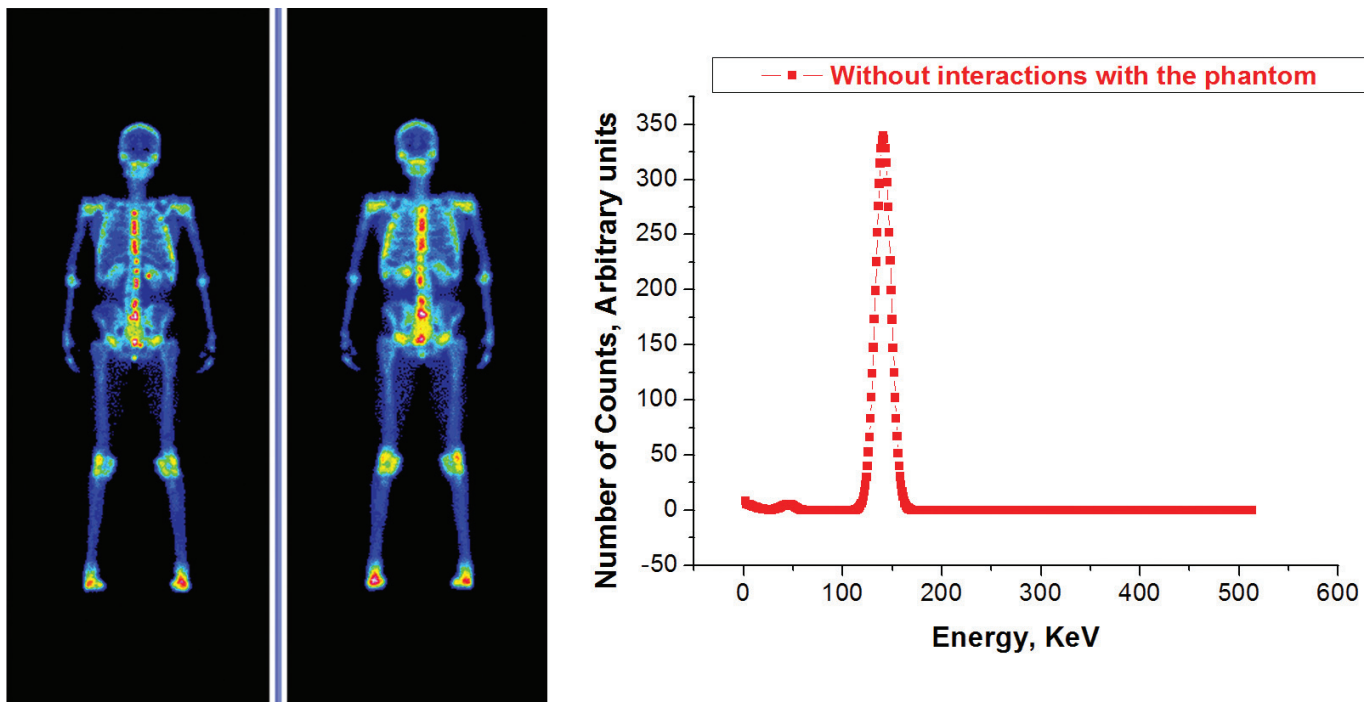

Figure 2. On the left, simulated images of the study without interactions with the phantom. On the right, the energy spectrum. LEHR collimator.
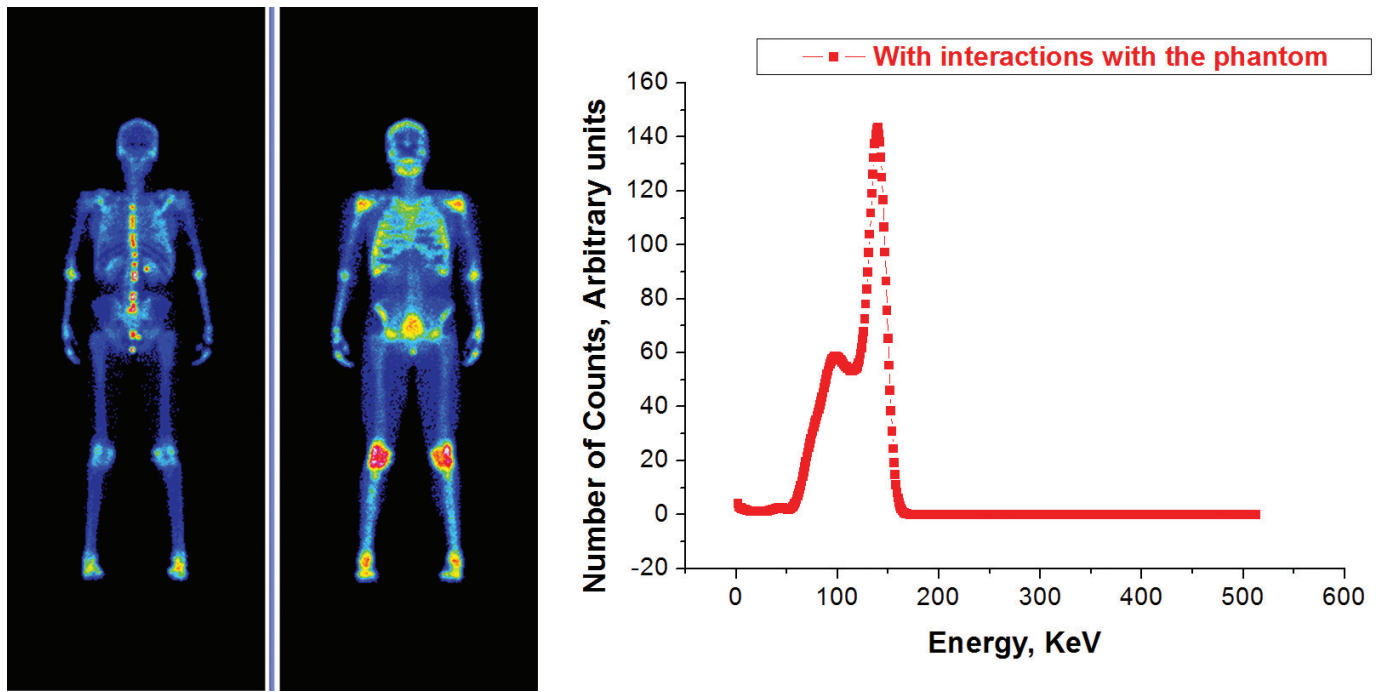

Figure 3. On the left, simulated images of the study with interactions with the phantom. On the right, the energy spectrum. LEHR collimator.

is observed that the images have blurring. Also, the lesions are not clearly observed on the phantom. If the scattered events are included in the image, the image will be a false representation of the source radioactive within the patient. Correction for scatter is most often made either in the energy domain where scatter in the photopeak energy window is modeled by collected data in additional energy windows or by using analytical methods that model the scatter on photopeak data directly (Khalil, 2011).

Figure 5 shows a comparison of the energy spectra of the three simulations. The first configuration shows a large photopeak. It is noticed that scattered radiation decreases the window fraction recorded with a photopeak window. It is appreciated a high Compton scattering spectra for the second and the third configuration. These spectra correspond to the phantom interactions and interactions with the backscattering material that simulates the dispersion in PMT. The differences between these simulations are not significant. The percentage error for Compton Area is only $2.41 \%$ and for Photo Area is $0.10 \%$.

Table 2 shows the parameters calculated by the SIMIND for each configuration.

The values in the table show that the number of scattering events is higher for configurations having interactions with the phantom and with backscattering material. The largest value corresponds to the last one 

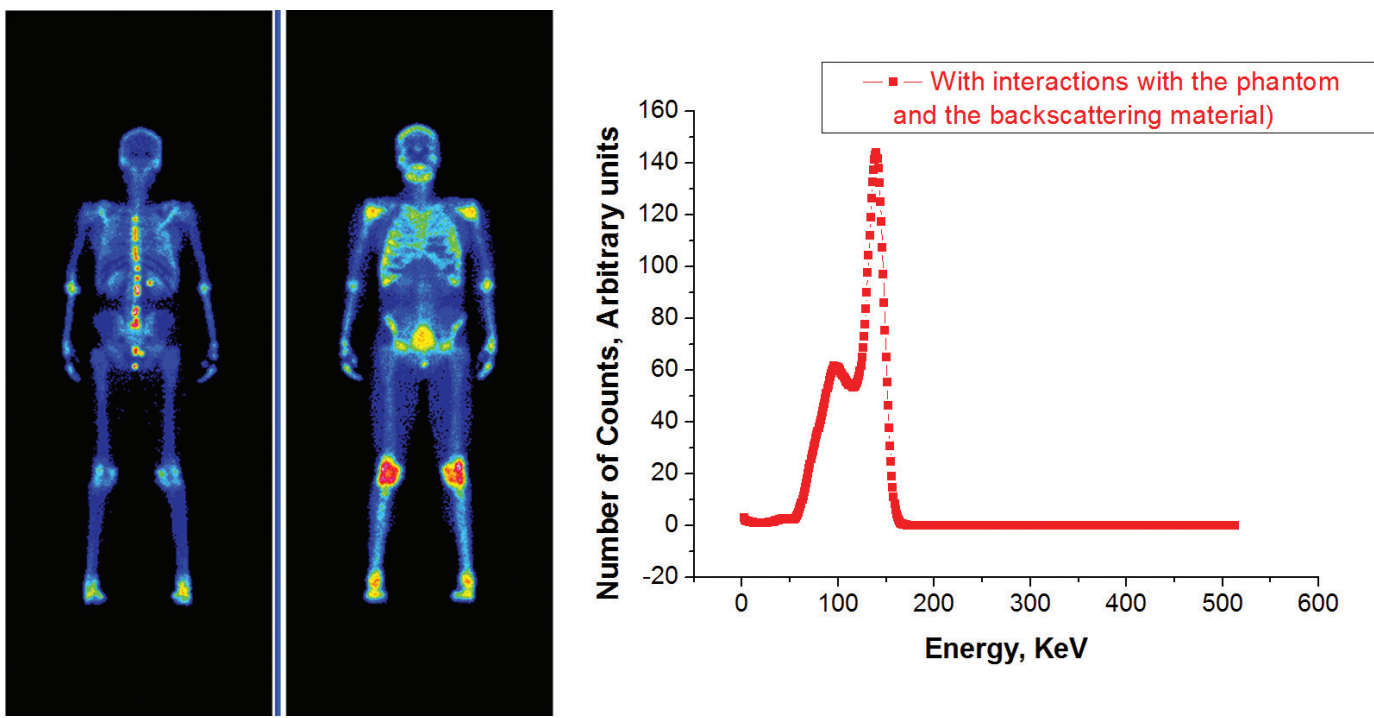

Figure 4. On the left, simulated images of the study with interactions with the phantom and the backscattering material. On the right, the energy spectrum. LEHR collimator.

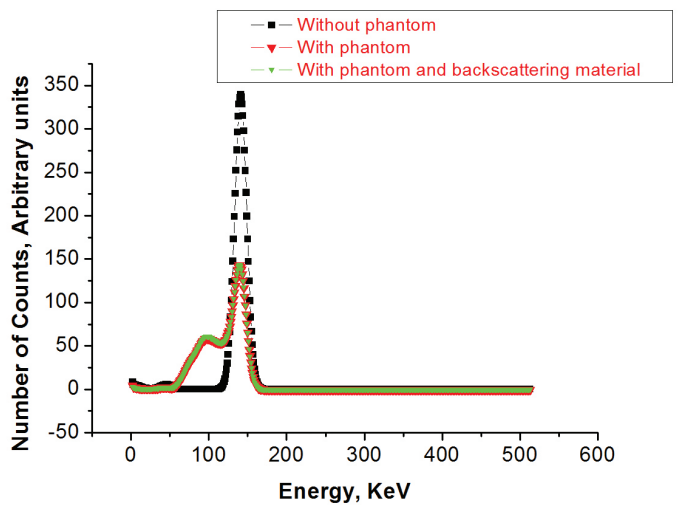

Figure 5. Comparison of the energy spectra of the three simulations.

because considers the contribution of more scattered events, but this is not significant for spectrum of ${ }^{99 \mathrm{~m}} \mathrm{Tc}$. The greatest number of events in the photopeak is observed in the first configuration. It does not take into account scattering events. The possibility of Pile Up is greater in the first configuration due to the increased contribution of the valid events in the energy window. The parameters involving scattering events are not evaluated in the first configuration. The fraction of events in the energy window that originates from Compton scattered photons is greater in the last configuration. It considers the contribution of more scattered events (detector scatter and object scatter). The values of the parameters: Scatter/Primary Ratio and Scatter/Total Ratio are higher for the last configuration. This is because Compton area and the contribution of photons scattered is higher for this configuration.
Also, the percentages of the scatter events originated from photons that have been scattered in the phantom more than once are higher for this configuration. The values of the parameters: Peak/Compton (Peak), Peak/Compton (Area) and Peak/Total are higher for the first configuration. This because the integral of simulated events between the lower energy window channel and the upper energy window channel is greater for this configuration.

The simulation corroborated that the images without influence of scattering events have a higher number of valid events recorded and improved the statistical quality of the image. The interactions of the gamma radiation produce few secondary photons which can contaminate the images.

The LEHR, LEUR, LEGP, LEHS, MEGP and HEHR collimators were simulated for the energy of $140 \mathrm{KeV}$, corresponding to ${ }^{99 \mathrm{~m}} \mathrm{Tc}$. Figure 6 shows the phantom Zubal images, taking into account the scattering in the phantom, scattering from the PMT and scattering from detector using LEHR and HEHR collimators.

Figure 7 shows the energy spectra corresponding to all collimators and Table 3 shows the parameters calculated by the SIMIND for each one of them.

From the generated images and the parameters calculated, it was confirmed that the area of the photo peak and the area of the Compton scattering are higher for LEHS collimator. This is because of its high sensitivity since this collimator is designed for operation at low energies. The low energy and high sensitivity collimators have thin septa. This condition allow them to pass a greater number of 
Table 2. Parameters calculated by the SIMIND for Zubal phantom simulations.

\begin{tabular}{|c|c|c|c|}
\hline Parameters & $\begin{array}{c}\text { No interactions with } \\
\text { phantom }\end{array}$ & $\begin{array}{c}\text { With interactions with } \\
\text { phantom }\end{array}$ & $\begin{array}{l}\text { With interactions with } \\
\text { backsattering material }\end{array}$ \\
\hline Compton Area (Spectrum) & $\begin{array}{c}392.5 \\
0.26 \%(1 \mathrm{SD})\end{array}$ & $\begin{array}{c}3069 \\
10.45 \%(1 \mathrm{SD})\end{array}$ & $\begin{array}{c}3145 \\
10.06 \%(1 \mathrm{SD})\end{array}$ \\
\hline Photo Area (Spectrum) & $\begin{array}{c}6128 \\
0.07 \%(1 \mathrm{SD})\end{array}$ & $\begin{array}{c}2811 \\
10.48 \%(1 \mathrm{SD})\end{array}$ & $\begin{array}{c}2814 \\
10.38 \%(1 \mathrm{SD})\end{array}$ \\
\hline Pileup Area (Spectrum) & $\begin{array}{c}232.7 \\
0.85 \%(1 \mathrm{SD})\end{array}$ & $\begin{array}{c}84.68 \\
13.57 \%(1 \mathrm{SD})\end{array}$ & $\begin{array}{c}84.74 \\
13.83 \%(1 \mathrm{SD})\end{array}$ \\
\hline Fraction Photo (Window) & $\begin{array}{c}1.0000 \\
0.07 \%(1 \mathrm{SD})\end{array}$ & $\begin{array}{c}0.9479 \\
11.25 \%(1 \mathrm{SD})\end{array}$ & $\begin{array}{c}0.9478 \\
11.15 \%(1 \mathrm{SD})\end{array}$ \\
\hline Fraction Compton (Window) & $\begin{array}{c}0.0000 \\
0.00 \%(1 \mathrm{SD})\end{array}$ & $\begin{array}{c}0.0521 \\
10.30 \%(1 \mathrm{SD})\end{array}$ & $\begin{array}{c}0.0522 \\
9.98 \%(1 \mathrm{SD})\end{array}$ \\
\hline Scatter/Primary Ratio & without scatter & $\begin{array}{c}0.3451 \\
7.58 \%(1 \mathrm{SD})\end{array}$ & $\begin{array}{c}0.3455 \\
7.67 \%(1 \mathrm{SD})\end{array}$ \\
\hline Scatter/Total Ratio & without scatter & 0.2566 & 0.2568 \\
\hline Percentage 1 st order & without scatter & $84.41 \%$ & $84.24 \%$ \\
\hline Percentage 2 nd order & without scatter & $13.89 \%$ & $14.03 \%$ \\
\hline Percentage 3 rd order & without scatter & $1.71 \%$ & $1.72 \%$ \\
\hline Peak/Compton (Peak) & 63.7682 & 57.8008 & 56.4121 \\
\hline Peak/Compton (Area) & 15.6099 & 0.9161 & 0.8948 \\
\hline Peak/Total & 0.9074 & 0.4713 & 0.4656 \\
\hline
\end{tabular}
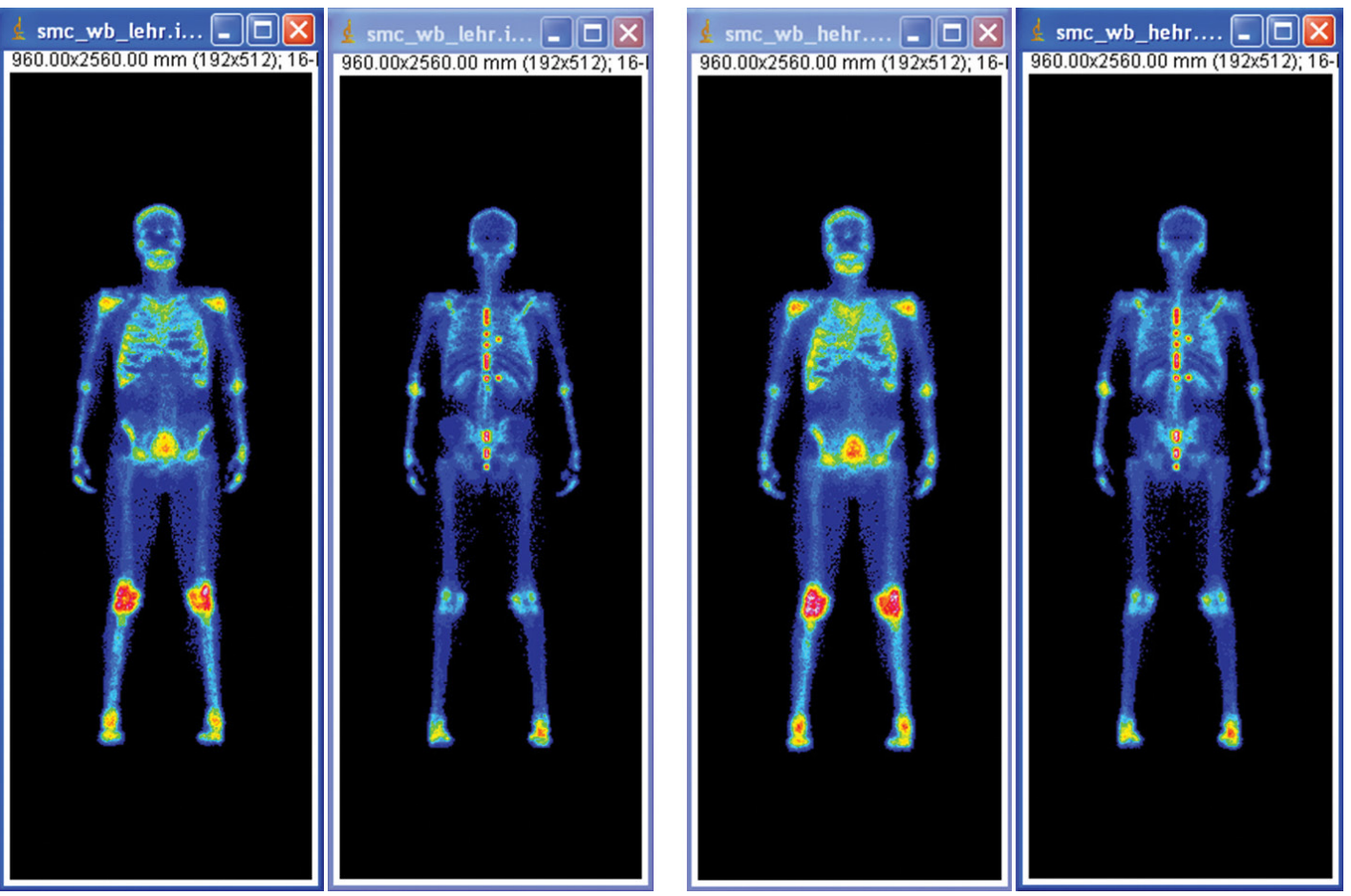

Figure 6. Images of the Zubal phantom considering the scattering in the phantom, scattering from the photomultipliers and scattering from detector for energy $140 \mathrm{KeV}$. Left: LEHR collimator. Right: HEHR collimator.

events at the expense of a poorer resolution. The LEHS collimator reported a higher contribution of scattered events. There is a loss of sensitivity for the HEHR collimator. This is due to its thickness $(61 \mathrm{~mm})$ since this collimator has high resolution but low sensitivity. It was designed for high energies. The length of septa is directly proportional to the energy. In addition minor events were recorded because the energy of $140 \mathrm{KeV}$ is absorbed by collimator.septas. When a high energy collimator is used with low 
Table 3. Parameters and detector energy spectrum calculated by the SIMIND for each simulation configuration of the Zubal phantom (LEHR, HEHR, LEUR, LEGP, MEGP and LEHS collimators for energy $140 \mathrm{KeV}$ ).

\begin{tabular}{lcccccc}
\hline \multicolumn{1}{c}{ Parameter } & LEHR & HEHR & LEUR & LEGP & MEGP & LEHS \\
\hline Compton Area & 3063 & 1344 & 2404 & 4498 & 9553 & $1345010.39 \%$ \\
(Spectrum) & $10.50 \%(1 \mathrm{SD})$ & $10.49 \%(1 \mathrm{SD})$ & $10.55 \%(1 \mathrm{SD})$ & $10.54 \%(1 \mathrm{SD})$ & $10.45 \%(1 \mathrm{SD})$ & $(1 \mathrm{SD})$ \\
Photo Area (Spectrum) & 2807 & 1231 & 2203 & 4127 & 8753 & 12340 \\
& $10.34 \%(1 \mathrm{SD})$ & $10.38 \%(1 \mathrm{SD})$ & $10.43 \%(1 \mathrm{SD})$ & $10.42 \%(1 \mathrm{SD})$ & $10.37 \%(1 \mathrm{SD})$ & $10.46 \%(1 \mathrm{SD})$ \\
Pileup Area (Spectrum) & 84.86 & 36.89 & 66.50 & 123.8 & 264.4 & 370.0 \\
& $13.68 \%(1 \mathrm{SD})$ & $13.48 \%(1 \mathrm{SD})$ & $13.59 \%(1 \mathrm{SD})$ & $13.32 \%(1 \mathrm{SD})$ & $13.93 \%(1 \mathrm{SD})$ & $13.84 \%(1 \mathrm{SD})$ \\
Fraction Photo & 0.9478 & 0.9477 & 0.9479 & 0.9478 & 0.9477 & 0.9476 \\
(Window) & $11.14 \%(1 \mathrm{SD})$ & $11.16 \%(1 \mathrm{SD})$ & $11.22 \%(1 \mathrm{SD})$ & $11.20 \%(1 \mathrm{SD})$ & $11.15 \%(1 \mathrm{SD})$ & $11.26 \%(1 \mathrm{SD})$ \\
Fraction Compton & 0.0522 & 0.0523 & 0.0521 & 0.0522 & 0.0523 & 0.0524 \\
(Window) & $10.14 \%(1 \mathrm{SD})$ & $10.38 \%(1 \mathrm{SD})$ & $10.53 \%(1 \mathrm{SD})$ & $10.42 \%(1 \mathrm{SD})$ & $10.78 \%(1 \mathrm{SD})$ & $10.27 \%(1 \mathrm{SD})$ \\
Scatter/Primary Ratio & 0.3467 & 0.3469 & 0.3463 & 0.3472 & 0.3464 & 0.3473 \\
& $7.50 \%(1 \mathrm{SD})$ & $7.69 \%(1 \mathrm{SD})$ & $7.49 \%(1 \mathrm{SD})$ & $7.62 \%(1 \mathrm{SD})$ & $7.58 \%(1 \mathrm{SD})$ & $7.52 \%(1 \mathrm{SD})$ \\
Scatter/Total Ratio & 0.2574 & 0.2575 & 0.2572 & 0.2577 & 0.2573 & 0.2578 \\
Percentage 1st order & $84.43 \%$ & $84.34 \%$ & $84.40 \%$ & $84.36 \%$ & $84.39 \%$ & $84.37 \%$ \\
Percentage 2nd order & $13.89 \%$ & $13.95 \%$ & $13.94 \%$ & $13.96 \%$ & $13.92 \%$ & $13.96 \%$ \\
Percentage 3rd order & $1.67 \%$ & $1.71 \%$ & $1.67 \%$ & $1.68 \%$ & $1.69 \%$ & $1.67 \%$ \\
Peak/Compton (Peak) & 60.0514 & 58.4461 & 59.5359 & 56.8927 & 58.5420 & 58.6012 \\
Peak/Compton (Area) & 0.9166 & 0.9161 & 0.9161 & 0.9174 & 0.9162 & 0.9170 \\
Peak/Total & 0.4714 & 0.4714 & 0.4713 & 0.4717 & 0.4713 & 0.4716 \\
\hline
\end{tabular}

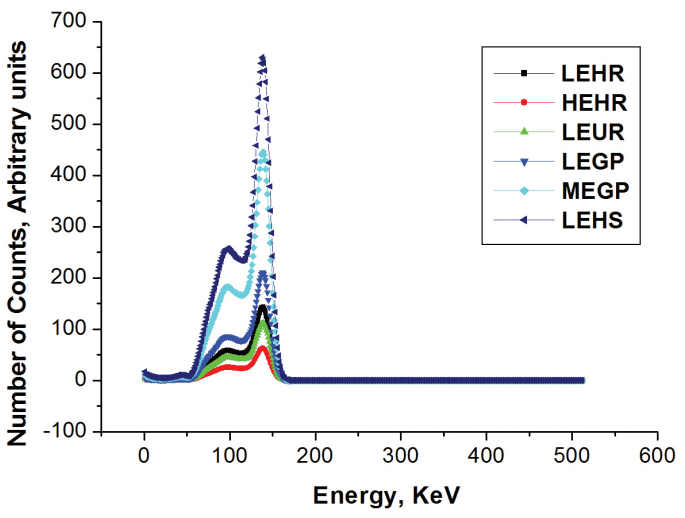

Figure 7. Energy spectra of the Zubal phantom for energy of $140 \mathrm{KeV}$. (LEHR, HEHR, LEUR, LEGP; MEGP and LEHS collimators).

energy photons, an image of normal quality will be obtained, but the camera will be working with less than best sensitivity.

The obtained results corroborated that particular attention should be paid to the use of collimators in clinics. It is necessary to take into account their sensitivity, energy range and purpose of each one of them.

\section{Discussion}

A simulation for evaluation of the scattered radiation components produced in gamma camera PARK using MC code SIMIND was made. The parameters related to this evaluation were calculated. The simulation confirmed that the interactions of the gamma radiation produce few secondary photons which can contaminate the images.

Comparisons among different collimators for the energy of $140 \mathrm{KeV}$ corresponding to ${ }^{99 \mathrm{~m}} \mathrm{Tc}$ was made. The parameters and detector energy spectrum were calculated. The simulation corroborated that LEHS collimator has higher sensitivity and HEHR collimator has lower sensitivity when they are used with low energy photons.

It was added the set of parameters for the gamma camera PARK collimators to the SIMIND database. This work can serve as a reference for future assessments of other gamma cameras. In addition it could be an aid for educational purposes and personal training of medical physics in nuclear medicine departments.

Another application of this work is quantification and correction for photon attenuation and scattering in nuclear medicine imaging. This is because of the ability of the MC code that separate detected events in their respective components.

\section{Acknowledgements}

I want to thank Dra Angelina Diaz Garcia of the Center of Technological Applications and Nuclear Development (CEADEN) and MsC Juan Osorio Deliz of the National Institute of Oncology and Radiobiology (INOR) in Havana, Cuba, for introducing me to the world of Medical Physics. 


\section{References}

Buvat I, Rodriguez Villafuerte M, Todd-Pokropek A, Benali H, Dipaola R. Comparative assessment of nine scatter correction methods based on spectral analysis using Monte Carlo simulations. Journal Nuclear Medicine. 1995; 36:147688. PMid:7629598.

De Vries DJ, King MA, Moore SC. Characterization of spectral and spatial distributions of penetration, scatter and lead X-rays in Ga-67 SPECT. IEEE Medical Imaging Conference Record. 1998; M9-6:1701-10.

Dewaraja YK, Ljungberg M, Koral KF. Characterization of scatter and penetration using Monte Carlo simulation in 131I imaging. Journal Nuclear Medicine. 2000; 41:12330. PMid:10647615 PMCid:PMC2811856.

Frey EC, Tsui BM. Use of the information content of scattered photons in SPECT by simultaneously reconstruction from multiple energy windows. Journal Nuclear Medicine. 1994; 35(5):17P.

Kadrmas DJ, Frey EC, Tsui BM. Application of reconstruction-based scatter compensation to thallium-201 SPECT: Implementations for reduced reconstructed image noise. IEEE Transaction Medical Imaging. 1998; 17:32533. PMid:9735896 PMCid:PMC2804953. http://dx.doi. org/10.1109/42.712122

Kadrmas DJ, Frey EC, Tsui BM. Simultaneous technetium99m/thallium-201 SPECT imaging with model-based compensation for cross-contaminating effects. Physics in Medicine and Biology. 1999; 44:1843-60. PMid:10442716
PMCid:PMC2808121. http://dx.doi.org/10.1088/00319155/44/7/319

Khalil MM. Basic sciences of nuclear medicine. Heidelberg, Dordrecht, London, New York: Springer-Verlag; 2011. http://dx.doi.org/10.1007/978-3-540-85962-8

Ljungberg M, King MA, Hademenos GJ, Strand SE. Comparison of four scatter correction methods using Monte Carlo simulated source distributions. Journal Nuclear Medicine. 1994; 35:143-51. PMid:8271036.

Rasband W, Ferreira TA. The ImageJ user guide. versión 1.43. 2010.

Sorenson JA, Cherry SR, Phelps M. Physics in nuclear medicine. 3rd ed. Saunders; 2002.

Sprawls P. Physical principles in medical imaging. 2nd ed. Madison: Medical Physics Publishing; 2000.

Wernick M, Aarsvold J. Emission tomography: The fundamentals of PET and SPECT. Elsevier Academic Press; 2004.

Zaidi H, Sgouros B. Monte Carlo calculations in nuclear medicine: Therapeutic applications. Taylor \& Francis; 2002. http://dx.doi.org/10.1201/9781420033250

Zaidi H. Quantitative analysis in nuclear medicine imaging. New York: Springer Science, Business Media, Inc.; 2006. http://dx.doi.org/10.1007/b107410

Zubal G, Harrell CR, Smith EO, Smith AL. Two dedicated software, voxel-based, anthropomorphic (torso and head) phantoms. Medical Physics. 1994; 21:299-302. PMid:8177164. http://dx.doi.org/10.1118/1.597290

\footnotetext{
Author 